\title{
El rol de la neuropsicología y la interdisciplinariedad en la etiología y neurofuncionalidad del Déficit de Atención e Hiperactividad
}

The role of neuropsychology and the interdisciplinarity in the etiology and Neurofunctionality of Attention-Deficit/Hyperactivity Disorder

O papel da neuropsicologia e da interdisciplinaridade na etiologia e Neurofuncionalidade do Transtorno por Déficit de Atenção com Hiperatividade

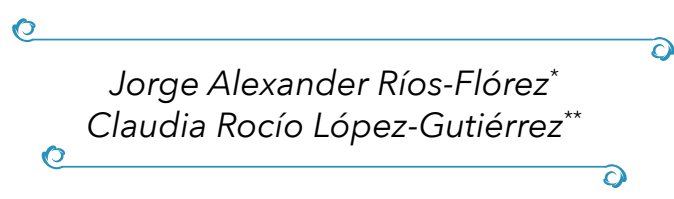

Recibido: 8.12.2017 • Arbitrado: 10.01.2018 •

Aprobado: 25.01.2018

Investigador-docente, Director del Grupo de Estudio e Investigación en Neurociencias Hippocampus; Magíster en neuropsicología Clínica; Miembro del Grupo Psicología, Educación y Cultura de la Institución Universitaria Politécnico Grancolombiano, Colombia. Doctorando en Psicobiología, Universidade Federal do Rio Grande do Norte, Brasil. Correspondencia: UFRN, Caixa Postal 1511 Campus Universitario, 59078-970, Laboratorio de Morfología, Centro de Biociências, Natal, RN-Brasil. E-mail: alexanderriosflorez@gmail.com

** Psicóloga-Investigadora, Coordinadora de la Línea de investigación en "Síndromes Neurológicos en la infancia" y la Línea de estudio en "Neurociencia afectiva y emocional" del Grupo de Estudio e Investigación en Neurociencias Hippocampus, Medellín, Colombia. E-mail: clarologu@hotmail.com

\section{Resumen}

El déficit de atención con hiperactividad es un trastorno caracterizado por la desatención y desinhibición conductual; así, puede tomar predominio desatento, hiperactivo-impulsivo y combinado o mixto, siendo la última la más frecuente. Son suficientes los estudios que confirman la presencia de signos biológicos en dicho síndrome, los genes transportadores y receptores de dopamina son los más implicados, igualmente se toma en cuenta el factor de heredabilidad. Para su diagnóstico se requiere exámenes neurológicos, así como la aplicación de pruebas neuropsicológicas y la utilización de otros métodos en psicología. En cuanto a la neuropsicología del TDAH en general, se presentan alteraciones en la atención selectiva y sostenida, en las funciones ejecutivas y en la memoria, con diferencias en los predominios. El déficit de atención con hiperactividad es de inicio temprano, y en algunos casos, persiste en la edad adulta, aunque con distintas manifestaciones. Por las áreas afectadas, el tratamiento para pacientes 
con TDAH debe ser multimodal, en la que se integre intervenciones neuropsicológicas, farmacológicas, psicosociales y psicopedagógicas, con el acompañamiento de sus familiares. Debe destacarse la intervención con programas de neuropsicología orientados a minimizar los compromisos funcionales de la actividad cerebral derivados del síndrome, abordándose de esta manera y no como un cuadro conductual de tipo trastorno.

Palabras clave: Atención, Híperactividad, Neuropsicología, Prefrontal, Psicopedagogía, TDAH.

\section{Abstract}

The Attention-Deficit/Hyperactivity is a disorder characterized by inattention and behavioral disinhibition; so, it can take predominance inattentive, hyperactive-impulsive and combined or mixed, being the last one the most frequent. Sufficient studies confirm the presence of biological signs in such syndrome, the dopamine transporter and receptor genes are the most involved and the heritability factor is also considered. For its diagnosis, neurological exams as well as the application of neuropsychological tests and the use of other methods in psychology are requested. As for the neuropsychology of ADHD in general, they are alterations in the selective and sustained attention in the executive functions and in the memory with differences in the predominance. Attention deficit hyperactivity disorder is early onset and in some cases, it persists in adulthood, although with different manifestations. Because of affected areas, treatment for patients with ADHD must be multimodal, in which neuropsychological, pharmacological, psychosocial and psychopedagogical interventions are integrated, with the family accompaniment. The intervention with neuropsychology programs aimed at minimizing functional commitments of brain activity derived from the syndrome, approaching in this way and not as a behavioral disorder-type case.

Keywords: Attention, Hyperactivity, Neuropsychology, Prefrontal, Psychopedagogy, ADHD.

\section{Introducción}

A lo largo de las últimas décadas ha sido creciente el número de investigaciones que se han centrado en el estudio de las alteraciones y cuadros clínicos que tienen como manifestación cuadros comportamentales acuñados a etiologías del neurodesarrollo, y que presentan repercusiones significativas en la cotidianidad de los individuos. Aún más, han surgido propuestas de investigación que ahondan en las comorbilidades cognitivas de estos déficits 
comportamentales a fin de comprender la relación entre cognición y conducta, todo con el objetivo de establecer propuestas de intervención encaminadas a subsanar en alguna medida los compromisos de la actividad cerebral derivados de tales cuadros clínicos. Este es el caso del trastorno por déficit de atención e hiperactividad (TDAH) que se caracteriza principalmente por un patrón persistente de comportamientos que reflejan desatención y desinhibición conductual no explicada por otro trastorno del pensamiento, desarrollo o de orden emocional (Capdevila-Brophy, Artigas-Pallarés y Obiols-Llandrich, 2006); inicia en la infancia, y es 3 a 4 veces más frecuente en niños que en niñas (Bayés et al., 2005; Muñoz, Palau, Salvadó y Valls, 2006; Ríos-Gallardo et al., 2017). Este cuadro clínico constituye el primer motivo de consulta en los centros paidopsiquiátricos, se presenta mundialmente, y sus manifestaciones se dan en forma continua, aunque variable, generando frecuentemente dificultades en el área escolar, familiar, social y laboral (Ruiz-García, Gutiérrez-Moctezuma, Garza-Morales y De la Peña-Olvera, 2005; Sánchez y Guillén, 2017; Martínez, Hernández, Durán, Lluch y Fitó, 2017; Fernandez-Mayoralas, Fernandez-Perrone, Munoz-Jareno y Fernandez-Jaen, 2017).

En la actualidad, el diagnóstico del TDAH se realiza con base a los criterios del Manual Diagnóstico y Estadístico de los trastornos mentales (DSM-5), donde es presentado en tres modalidades, con predominio inatento, hiperactivo-impulsivo y mixto o combinado (APA, 2013; Ruiz-García et al., 2005). Vale la pena anotar que el término hiperactividad se introdujo en el DSM-II en 1968 y el de alteración por déficit de atención en el DSM-III en 1980 (Muñoz et al., 2006); el abordaje del Trastorno a lo largo de las diferentes versiones del DSM ha sido controversial y ampliamente criticado.

Así, y clarificando sus bases conceptuales, la inatención se refiere a la incapacidad que tiene una persona para concentrarse por los periodos de tiempo requeridos para culminar una actividad que requiera un esfuerzo mental sostenido (Mateo y Vilaplana, 2007). De igual forma, la hiperactividad es la actividad motora exagerada, razón por la cual los pacientes tienen dificultad para mantenerse quietos, por lo que mueven regularmente los pies y las manos, y cambian constantemente de posición (Romero-Ayuso, Maestú, González-Marqués, Romo-Barrientos y Andrade, 2006). Por otra parte, la impulsividad básicamente se presenta como la dificultad que tienen los pacientes para lograr el autocontrol (Solis y Quijano, 2014). Por último, la modalidad mixta o combinada del Trastorno hace que estos pacientes tengan gran dificultad para desenvolverse en su medio con naturalidad, ya que la severidad de los síntomas de inatención, impulsividad e hiperactividad es similar entre sí. Estadísticamente, la presentación más común del TDAH es la mixta o 
combinada, seguida del tipo inatento y, por último, la hiperactivo-impulsivo (Ruiz-García et al., 2005). En relación a esto último, se encontró una mayor prevalencia en el género masculino para los subtipos hiperactivo-impulsivo y mixto, y el subtipo inatento para el género femenino (Van, Gutiérrez, González y Ibáñez, 2008).

\section{Aportes a la etiología del TDAH}

Los estudios actuales, llevados a cabo en áreas como la genética, neuroquímica, morfometría y principalmente y en la neuroimagenología, aportan información suficiente para apoyar la base biológica del déficit de atención con hiperactividad. En este sentido, la biología molecular ha reconocido dos genes relacionados con este trastorno, el gen transportador de dopamina (DAT) y el gen receptor de dopamina (DRD4). También se han considerado los genes reguladores de la noradrenalina y de la nicotina (Ruiz-García et al., 2005). Los hallazgos de los genes implicados en este trastorno se han identificado principalmente a partir de la respuesta a los tratamientos farmacológicos (Bayés et al., 2005).

Por otra parte, Solovieva, Lázaro y Quintanar (2008), han sugerido una debilidad en los lóbulos frontales, especialmente en el área premotora y prefrontal, pues no alcanzan un desarrollo funcional adecuado en niños con TDAH. También se han sugerido un tamaño menor relacionado a los ganglios basales particularmente en el putamen de ambos hemisferios y del globo pálido derecho (Mulas et al., 2012). Igualmente, estudios con gemelos revelan una heredabilidad aproximada del 76\% en el TDAH (Mulas et al., 2012; Muñoz et al., 2006).

Lo anterior, se ha referido en relación a la etiología neuroanátomo-fisiológica del TDAH en general, pues estudios recientes identifican una etiología diversa por tipos, así el TDAH con predominio de déficit de atención, implica una mayor disfunción anterior del lóbulo frontal, lo que genera una alteración en la atención sostenida y en los mecanismos de control inhibitorios corticales; y en el predominio de hiperactividad-impulsividad se presenta una mayor disfunción neurológica posterior, especialmente en el hemisferio derecho, condicionando una alteración en la atención selectiva, trastornos de psicomotricidad, deficiente manejo de la anticipación y control de impulsos (Mulas et al., 2012; Muñoz et al., 2006). Por último, mientras es común relacionar el consumo de azúcar con el TDAH, diversos autores no encuentran una asociación directa y los estudios no son concluyentes al respecto (Sáenz, 2005; Pacheco, 2015). 


\section{Abordaje diagnóstico del TDAH}

El diagnóstico del TDAH es netamente clínico, y los casos deben ser cuidadosamente estudiados, pues aproximadamente el 65\% de los pacientes tienen un trastorno comórbido (Ruiz-García et al., 2005). Igualmente, es importante anotar que la sintomatología debe estar presente en los diferentes contextos en los que se desenvuelve el sujeto -social, académico, laboral- (APA, 2013). Al mismo tiempo, hay que tener en cuenta que para realizar el diagnóstico del TDAH en una persona adulta es necesario que el trastorno esté presente desde la infancia, como mínimo desde los 7 años; por lo que se requiere integrar todos los datos clínicos disponibles (Ramos-Quiroga et al., 2006).

Además, la aplicación de pruebas resulta bastante importante para confirmar la presencia de las características clásicas de la neuropsicología del TDAH (Etchepareborda, Paiva-Barón y Abad, 2009). Los test neuropsicológicos más usuales en la exploración del TDAH son el Continuous Performance Test -CPT (Conners y Sitarenios, 2011), que permite evaluar la atención sostenida y la capacidad de control motor, el Test de Stroop (Golden, 2001), que permite evaluar el control inhibitorio de interferencia frente a estímulos con doble input y el Test de selección de cartas de Wisconsin -WCST (Grant y Berg, 2001), que evalúa la flexibilidad cognitiva, entre otros.

De igual forma, la neuroimagen proporciona ciertos elementos claves para su diagnóstico, como la tomografía computarizada, la resonancia magnética y técnicas de neuroimagen funcional (Mulas et al., 2012). De esta manera, su evaluación debe realizarse por medio del método observacional neuroconductual, formado por un equipo multidisciplinario (Muñoz et al., 2006). Así mismo, se presenta la importancia de realizar un diagnóstico diferencial del TDAH, frente a trastornos de lectura y escritura (Mejía y Varela, 2015; Ruiz y Sicachá, 2015), incluso frente al trastorno del espectro autista (Berenguer, Miranda, Pastor y Roselló, 2016; Rico-Moreno y Tárraga-Mínguez, 2016).

\section{Funcionamiento Neuropsicológico en el TDAH}

Con relación a la Neuropsicología del síndrome en general, Etchepareborda, Paiva-Barón y Abad (2009), Arán y Mías (2009) y Galindo y Valera (2014), refieren deficiencias principalmente en la atención selectiva y sostenida y en las funciones ejecutivas. López-Campo, Gómez-Betancur, Aguirre-Acevedo, Puerta y Pineda (2005), concluyen en su estudio alteraciones en el esfuerzo cognitivo, vigilancia continua y la organización verbal fonológica y semántica. Además, se encuentran disfunciones en las tareas de secuencia gráfica, con 
intrusiones y perseveraciones (Solovieva, Lázaro y Quintanar, 2008; Román, 2014). Igualmente, la memoria es una función seriamente afectada, en las modalidades verbal, visual, procedimental y de trabajo, aunque en distintas proporciones y con diferencias entre pruebas neuropsicológicas (Bará-Jiménez, Vicuña, Pineda y Henao, 2003; Martín-González et al., 2008; Roselló y Berenguer, 2016)

En cuanto a la Neuropsicología del TDAH por tipos, López-Campo et al. (2005) observaron en su estudio una ejecución significativamente menor en las pruebas de fluidez verbal para el predominio hiperactivo-impulsivo, además de un déficit en la memoria inmediata y diferida; y por supuesto, un mayor déficit en el control inhibitorio de conductas impulsivas (Arán y Mías, 2009; Ramos y Pérez-Salas, 2017). En los niños con predominio inatento, la velocidad del procesamiento de la información se encuentra especialmente afectada (Arango, Uribe, Cardona y Cornejo, 2008), sumada a la disminución de la fluidez semántica (Capdevila-Brophy et al., 2005). Así mismo, Arán y Mías (2009) hallaron una deficiencia en la memoria verbal inmediata y diferida, con más frecuencia de perseveraciones, lo que da cuenta de una menor flexibilidad cognitiva, a parte de la evidente alteración en la atención sostenida y selectiva. Sin embargo, no se reportaron alteraciones significativas en la figura de Rey para ninguno de los dos grupos (clínico y control), por lo que se puede concluir que la memoria en el TDAH mejora su desempeño tras la presencia de un apoyo visual (Arán y Mías, 2009).

Por otra parte, Capdevila-Brophy et al. (2005), han encontrado que el TDAH en su modalidad mixta o combinada presenta un menor desempeño en lo referente a su neuropsicología, especialmente en comparación con el tipo inatento, en la memoria de trabajo no verbal, control motriz y algunos componentes de la función ejecutiva; sin embargo, estos autores destacan una mejor habilidad en la composición gestáltica.

Asimismo, es interesante destacar el hallazgo de Solovieva, Lázaro y Quintanar (2008), el cual señala diferencias neuropsicológicas en algunos elementos, entre niños con TDAH y niños procedentes de escuelas rurales, y no con niños de procedencia urbana, lo que habla de la fuerte influencia cultural en la función cognitiva, una conclusión igualmente descrita por Carrasco et al. (2012).

Entre otras particularidades, Ríos-Flórez, Álvarez-Londoño, David-Sierra y Zuleta-Muñoz (2018), describen una relación entre el TDAH y la condición de prematurez, en donde el $7.5 \%$ de los niños prematuros presentaron TDAH tipo combinado, $10 \%$ el tipo inatento y el $33.75 \%$ tipo hiperactividad-impulsividad, lo que trae consigo, el desarrollo de conductas, emociones y actitudes 
que repercuten de manera negativa en el entorno de los niños, lo anterior, teniendo en cuenta, la implicación a nivel cerebral del nacimiento pre-término, especialmente en la maduración y funcionamiento de los lóbulos frontales.

\section{TDAH en adultos}

El TDAH se inicia en la infancia y en más del 50\% de casos, persiste también en la edad adulta (Ramos-Quiroga et al., 2006), por lo que se han encontrado investigaciones que tratan de realizar un bosquejo del perfil neuropsicológico del adulto con déficit de atención e hiperactividad. Las funciones ejecutivas han sido uno de los procesos cognitivos más discutidos, así Rodríguez-Jiménez et al. (2006) plantean alteraciones en la respuesta de inhibición, capacidad de planificación, flexibilidad cognitiva, memoria de trabajo y fluidez verbal, dificultades similares a las de niños con el mismo trastorno. Igualmente, se dan fenómenos patológicos como las omisiones, predecibles en cierta medida por las dificultades en la atención y los errores de comisión por la dificultad en la inhibición de respuestas, explicado por la impulsividad presente.

Pese a lo anterior, la sintomatología del TDAH experimenta ciertos cambios en los adultos, así, la hiperactividad es el síntoma que más se reduce, seguido de la impulsividad, y la inatención se mantiene prácticamente igual, razón por lo cual, el TDAH se asocia a problemas académicos, laborales, legales, familiares y a la presencia de otras patologías psiquiátricas (Ramos-Quiroga et al., 2006).

En la mayoría de los casos, los síntomas del TDAH en adultos pasan desapercibidos, enmascarándose en conductas y roles aceptables socialmente o en profesiones y actividades que incentivan su presencia, lo que dificulta su identificación y manejo. Clínicamente acuden a consulta aquellos adultos en que su quehacer diario exige mayor control comportamental y mayores esfuerzos cognitivos, por lo que sólo a través de pruebas neuropsicológicas es posible dar cuenta de la presencia de TDAH en adultos, más allá de evaluar únicamente su conducta y las interferencias de los síntomas en la cotidianidad; un adecuado rastreo y desarrollo de la historia clínica facilitará el abordaje y manejo del síndrome en esta población.

\section{TDAH y Emociones}

Como se ha podido evidenciar, el TDAH tiene un amplio abordaje a nivel cognitivo, sin embargo, es substancial rescatar las implicaciones que se generan con relación a los componentes afectivo, emocional y motivacional 
(López-Martín, Albert, Fernández-Jaén y Carretié, 2010; Campeño-Martínez, Gázquez-Linares y Santiuste-Bermejo, 2016). Específicamente, se ha encontrado que "tanto niños como adultos con TDAH presentan una disfunción primaria en el reconocimiento de los estímulos emocionales (expresiones faciales emocionales y prosodia afectiva) y una importante discapacidad para modular sus emociones, especialmente cuando éstas son negativas" (Albert, López-Martín, Fernández-Jaén y Carretié, 2008, p.39). Una condición desfavorecedora, si se tiene en cuenta que esto constituye un factor de riesgo para la difusión de dificultades en el ajuste escolar, social y relacional, y por supuesto a nivel personal, en tanto se da una mayor inestabilidad y explosión emocional (Guerrero y Pérez, 2011; Sánchez-Pérez y González-Salinas, 2013). Igualmente, se presenta una fuerte congruencia cuando se enuncian los dos síntomas secundarios más frecuentes en este trastorno, como lo son la baja tolerancia a la frustración y la baja tolerancia al esfuerzo (Orjales, 2007).

A pesar de lo anterior, se ha documentado un aumento de la adaptación emocional tras un proceso de intervención psicosocial, como lo afirman en su estudio Presentación, Siegenthaler, Jara y Miranda (2010), que, además, contó con variables como la adaptación académica y social. Adicional a esto, es importante destacar el tema de la teoría de la mente (ToM), donde una investigación realizada en Colombia, que evaluó tres componentes de la misma en niños con TDAH, a saber, las atribuciones de falsas creencias, el lenguaje pragmático y las atribuciones a la mirada, encontró un rendimiento significativamente inferior en las 2 primeras tareas, más no en la última, lo que podría presentarse como un factor explicativo de la dificultad en las habilidades sociales descritas anteriormente (Pineda y Puentes, 2013); resultados corroborados por Orozco y Zuluaga (2015), que relatan diferencias en las tareas de atribuciones de falsas creencias de primer y segundo orden, entre niños diagnosticados con TDAH y un grupo control. En este mismo sentido, tienen consonancia los hallazgos de Miranda-Casas, Baixauli-Fortea, Colomer-Diago y Roselló-Miranda (2013, p. 177), quienes afirmaron que "la presencia de síntomas de TDAH en individuos con un diagnóstico clínico de autismo es muy elevada".

\section{Tratamientos emergentes para el TDAH}

El tratamiento para el TDAH en caso de recurrir a la farmacología debe estar acompañado de neuropediatría, psiquiatría y paidopsiquiatría (Ruiz-García, Gutiérrez-Moctezuma, Garza-Morales y De la Peña-Olvera, 2005). Desde este enfoque, la medicación psicoestimulante es el tratamiento más utilizado (Arco, Fernández e Hinojo, 2004), sin embargo, la atomoxetina, el primer 
fármaco no psicoestimulante, ha demostrado su eficacia en el tratamiento de este trastorno para niños y adultos (Ramos-Quiroga et al., 2006). A pesar de los efectos positivos a corto plazo del tratamiento farmacológico, su uso está rodeado por una gran controversia por la posible aparición de efectos secundarios (Arco, Fernández e Hinojo, 2004).

Para mayor efectividad, el tratamiento con medicamentos debe integrarse al psicosocial, con la participación de padres de familia, psicólogos, neuropsicólogos y docentes, teniendo claramente en cuenta la particularidad del caso, pues es necesario recordar las disfuncionalidades que trae consigo este trastorno en la cotidianidad, principalmente a nivel escolar, laboral y social. El abordaje desde la psicología conductual ya sea individual o grupal, ha sido la de mayor éxito, por lo que, obtener información acerca de las estrategias de afrontamiento de los síntomas por parte del paciente y su núcleo más cercano puede resultar bastante útil (Ruiz-García et al., 2005), para prever conductas, su identificación oportuna y abordaje.

Al respecto, Mulas et al. (2012), resaltan las asesorías psicopedagógicas como un elemento fundamental en la intervención del TDAH, pues brindan entrenamiento a los padres y profesores, en el manejo con sus hijos y sus estudiantes. Sin embargo, en las actividades psicopedagógicas están en mayor medida involucrados los docentes, pues es en el contexto escolar, donde se hacen más evidentes las manifestaciones del síndrome, de esta manera, ellos pueden situar al alumno cerca de ellos, con el fin de generar una mayor interacción, de estar más pendientes de su comportamiento y preguntarles con frecuencia elementos en relación a la clase, darles más tiempo en la resolución de los exámenes o modificando la forma de presentación, para que puedan mejorar su rendimiento académico y, por ende, su autoestima. Los autores citados, proponen un modelo de intervención Cognitivo-Conductual en el que se realizan formaciones a padres y profesores en condicionamiento, especialmente operante, con el fin de modificar conductas para lograr una mayor adaptación al medio, junto con la enseñanza al niño de técnicas de autocontrol y resolución de problemas.

Lo anterior, lo refuerzan autores como De la Peña et al. (2009), los cuales afirman que las intervenciones más eficaces en el TDAH son aquellas llamadas multimodales, en las que se integran medidas farmacológicas y psicosociales, incluyendo intervenciones familiares, escolares y conductuales. Además, se ha propuesto una serie de etapas en el procedimiento de intervención, en la etapa uno (1) se considera la psicoeducación básica, en la etapa dos (2) es recomendable el uso de estimulantes como el metilfenidato o anfetaminas, para la tres (3) el uso de la desipramina, la etapa cuatro (4) incluye la opción 
de los agonistas alfa adrenérgicos y la última etapa (5) requiere que un clínico realice una propuesta de combinación o adición de otros procedimientos. A lo anterior debe sumarse la evaluación neuropsicológica desde el inicio y el desarrollo e integración de un programa de rehabilitación longitudinal a estas etapas, a fin de promover mejorías en las funciones cognitivas comprometidas, e incentivar el retraso y/o evolución de procesos neuropsicológicos previsibles de ser alterados en el curso de la patología.

\section{El síndrome y la intervención Neuropsicológica; Aspectos}

\section{de análisis}

El déficit de atención e hiperactividad ha sido uno de los cuadros clínicos infantiles que más se ha diagnosticado desde la última década del siglo pasado, sin embargo, aun cuando ha recibido amplio abordaje en sus manifestaciones sintomáticas y la clínica funcional subyacente, los correlatos neuroanatómicos y fisiológicos han sido diversos y debatidos en diversos estudios, por lo que llegar a un consenso sobre tales correlatos no ha sido fácil ni definitivo, considerando la relevancia que acarrea el integrar la neuroendocrinología para este objetivo. Si bien los estudios que describen las regiones cerebrales asociadas al TDAH no son concluyentes, una zona de convergencia sobre la actividad del cuadro clínico está referida a los lóbulos frontales en consideración a los productos de actividad cerebral atribuidos a estas estructuras filogenéticamente recientes en la evolución de la especie humana, aun cuando ontogenéticamente tienen un desarrollo estructural y fisiológico tardío, abarcando para esto un cuarto de la vida del individuo. Así, las manifestaciones de alteración funcional que caracterizan al TDAH son fallos de carácter cognitivo-comportamental que aparecen mucho antes de la estructura cerebral alcanza su "desarrollo final", el cual está cerca de los 21 años de edad, momento en el que los procesos de mielinización alcanzan el polo más anterior del encéfalo. En consideración a lo anterior, y tomando en cuenta la presencia de los síntomas del trastorno desarrollados desde los primeros 6 años de vida como requisito fundamental, es posible inferir y plantear que el TDAH se presenta como una manifestación metabólica netamente asociada a la química cerebral y a la fisiología requerida para esto, en este sentido, no es viable afirmar que este trastorno obedezca a malformaciones estructurales o errores de neurogénesis y migración celular, por lo que sus manifestaciones clínicas dependerán de la actividad neuronal y las interacciones de conectividad que éstas establezcan a lo largo del tejido cerebral, así como del transporte electroquímico de sus procesos neurofisiológicos, destacándose los 
lóbulos prefrontales como el centro donde se orquesta e integra la regulación cognitivo-comportamental.

Dentro de los múltiples debates que ha suscitado el TDAH, la mayor crítica recibida está en torno al sobrediagnóstico y los errores cometidos para establecerlo. En la mayoría de los casos los niños y niñas llegan a consultar al especialista con la etiqueta de "TDAH" otorgada por sus profesores u orientadores de las escuelas y colegios que frecuentan; y aun cuando son estas las personas idóneas para identificar en primera instancia aquellos comportamientos catalogados como clínicos, no lo son para asignar el diagnóstico y esto no obedece a su rol profesional, sino a la falta de preparación e instrucción que los caracteriza para abordar las manifestaciones del cuadro clínico. El TDAH se ha tornado una etiqueta popular para catalogar a aquellos individuos que conductualmente sin "inquietos" y desarrollan varias tareas en un mismo momento o que quieren experimentar "de todo un poco", y también se ha atribuido erróneamente a niños y niñas que dentro de periodos de crecimiento y desarrollo habitual se espera como obvio que tengan conductas hiperactivas, sin embargo, el entorno donde tales manifestaciones se expresan ha estado determinando arbitrariamente si estos son aceptados como "normales" o no, e infortunadamente fuera de los entornos escolares y familiares, y llegando a los consultorios de atención especializada, los profesionales clínicos tienen un amplio margen de error sobre la pericia para determinar y analizar si realmente el sujeto presenta o no TDAH.

Los diagnósticos han sido usualmente otorgados por psicólogos, y dentro de estos, la consideración para establecerlo redunda en lo que sea establecido por el DSM, así, determinar el diagnóstico se ha tornado como una simple tarea en la cual se cumpla una lista de requisitos sin llegar a analizar a profundidad las relaciones sintomáticas, la valoración de los diversos entornos sobre los que se desarrolla y desenvuelve el individuo y mucho menos se llegan a considerar los determinantes neurobiológicos del TDAH; esto, debido a la falta de conocimiento e instrucción de quién atiende la consulta. De otro lado, la principal consideración que debe hacerse sobre los criterios diagnósticos del TDAH recae sobre su tipología como condición patológica. Ha sido un error abordar las manifestaciones alteradas de la actividad cerebral como un Trastorno, puesto que en sí misma la tipología de trastorno es inflexible ante la expresión clínica de los síntomas considerados para cualquier condición neuropsiquiátrica, como simple ejemplo el "TDAH" como trastorno y desde sus criterios diagnósticos no puede ni debe establecerse a los 5 años de edad del sujeto, pese a estar claras las alteraciones cognitivo-comportamentales asociadas a tal desregulación encefálica, por lo que la clínica funcional y la 
neurobiología del déficit de atención e hiperactividad debe plantearse como un Síndrome y no como un trastorno, puesto que el síndrome es flexible en la presencia de los síntomas, considera diferentes momentos de aparición de cada uno de estos y analiza de forma adecuada la evolución del cuadro clínico al vincular y correlacionar la etiología, los mecanismos biológicos subyacentes y el curso de las manifestaciones cognitivo-comportamentales; pudiéndose clasificar como un síndrome de predominancia frontal. Lo anterior favorece ampliamente el abordaje interdisciplinar que se requiere para dar manejo y control al cuadro clínico, puesto que la conceptualización e integración adecuada del mismo determinará y orientará fundamentalmente la intervención necesaria.

\section{Interdisciplinariedad y estudios futuros}

En relación con el abordaje clínico del síndrome por déficit de atención e hiperactividad, es claro que se requiere un abordaje interdisciplinar, puesto que se irradia como un cuadro clínico bio-psico-social. En este sentido, es clave la intervención por psicología para el trabajo conjunto entre el entorno educativo/laboral, familiar y social, al punto de lograr establecer el correcto manejo y regulación de los estados emocionales tanto inherentes al individuo como de sus interacciones relacionales en estos entornos. De otro lado, la valoración por psiquiatría es crucial, no para determinar la inmediata implementación de fármacos, como ha sido usual, sino para integrarse al programa de psicología y monitorear el curso y progreso del tratamiento conductual para, eventualmente considerar o descartar el inicio de terapias farmacológicas (ya que es claro que no todos los casos requieren medicación), las cuales deben ser inversamente proporcionales a los progresos positivos alcanzados por psicología. Hasta aquí este ha sido el abordaje usual de las manifestaciones clínicas del síndrome, pese a esto, debe ser posible llegar a consultar neuropediatría, pedagogía y psicología educativa, sin embargo, aun cuando éstas se vinculen, es frecuente que se dejen de lado las repercusiones cognitivas asociadas (descritas en apartados anteriores), por lo en la mayoría de los casos se logran progresos en control conductual y mínimos en procesos atencionales, lo que acelerará los fallos cognitivos e intensificará las comorbilidades, como las dificultades del aprendizaje, entre otros; es por esto, que la batuta del trabajo interdisciplinar a desarrollarse, debe estar en cabeza del neuropsicólogo clínico.

Cuando se planea la importancia de la intervención neuropsicológica, debe pensarse como un mecanismo para habilitar aquellas funciones cognitivas que no llegaron a expresarse en el nivel esperado, a su vez, rehabilitar aque- 
llas que por carencia de estimulación o por influencia de factores adyacentes decayeron, por lo que se impulsa su recuperación, y fundamentalmente estimular la actividad cerebral a fin de potencializar procesos no comprometidos e incentivar el desarrollo de habilidades compensatorias que favorezcan ampliamente el hándicap del paciente. Lo anterior requiere que el neuropsicólogo no centre únicamente sus esfuerzos en un programa cognitivo, sino que integre las emociones del sujeto, sus fortalezas personales, familiares y sociales, y busque adaptarlo a su entorno de la mejor manera posible, a partir de la regulación de los productos de la actividad cerebral, y considerando la individualidad del sujeto al formular los programas de intervención, así como el subtipo/predominio presentado (hiperactivo, impulsivo, combinado). Es en este punto de la intervención donde la interdisciplinariedad emerge, el profesional en neuropsicología no debe acaparar ni pretender extender su labor a la de otras áreas y profesionales, por el contrario, debe interconsultar estas otras especialidades, que desde cada óptica enriquecerán y aportarán a los progresos y revaloraciones de la evaluación e intervención neuropsicológica que serán requeridas para tratar niños, niñas, adolescentes y/o adultos con síndrome por déficit de atención e hiperactividad; logrando así, extrapolar y extender a entornos pedagógicos, laborales y sociales, las actividades de los programas de intervención neuropsicológica, implementándose indirectamente como mecanismo de evaluación del programa en los entornos donde realmente es necesario que el individuo se desenvuelva y, con las herramientas obtenidas, regule adecuadamente su actividad cognitivo-comportamental.

De esta manera, la intervención debe ser farmacológica (en caso de requerirse), psicosocial, psicopedagógica y de rehabilitación cognitiva, con la indispensable implicación de las personas que constituyen el núcleo más cercano del paciente. Es por lo anterior, que día a día crece el interés por el estudio del síndrome desde las diferentes áreas de la ciencia médica y psicológica, lo que da lugar a que se desarrollen diversos y adecuados enfoques de intervención. Sin embargo, y a pesar de los avances científicos, es necesario seguir ahondando en la neuroanátomo-fisiología del cuadro clínico, y en la formación de profesionales idóneos que comprendan ampliamente la actividad cerebral, con el fin de desarrollar nuevas y eficaces estrategias que permitan establecer fiable y oportunamente el diagnóstico, ante el cual se diferencie la modalidad predominante, con el ánimo de idear una intervención acorde a la naturaleza presentada. Así mismo, deben surgir nuevas propuestas de investigación que apunten a mejorar los tratamientos farmacológicos en pro de reducir su dependencia y los efectos adversos asociados sobre la cognición y el comportamiento. 


\section{Referencias}

Albert, J., López-Martín, S., Fernández-Jaén, A., Carretié, L. (2008). Alteraciones emocionales en el trastorno por déficit de atención/hiperactividad: datos existentes y cuestiones abiertas. Revista de Neurología, 47(1): 39-45. Recuperado de https:// www.neurologia.com/articulo/2008111

Arango, L., Uribe, M., Cardona, J., Cornejo, J. (2008). Características clínicas, neuropsicológicas y sociodemográficas de niños varones con déficit de atención/ hiperactividad de tipo inatento en Medellín, Antioquia, Colombia 2004-2005. Iatreia, 21(4): 375-385. Recuperado de http://www.scielo.org.co/scielo. php?pid $=$ S0121-07932008000400003\&script $=$ sci_arttext

Arán, V., Mías, C. (2009). Neuropsicología del Trastorno por Déficit de Atención/ Hiperactividad: subtipos predominio Déficit de Atención y predominio HiperactivoImpulsivo. Revista Argentina de Neuropsicología, 13: 14-28. Recuperado de http:// www.revneuropsi.com.ar/pdf/numero13/AranyMias.pdf

Arco, J., Fernández, F., Hinojo, F. (2004). Trastorno por déficit de atención con hiperactividad: intervención psicopedagógica. Psicothema, 16(3): 408-414. Recuperado de http://www.redalyc.org/articulo.oa?id=72716312

Asociación Americana de Psiquiatría. (2013). Manual Diagnóstico y Estadístico de los trastornos mentales (DSM-5). Madrid: Editorial Médica Panamericana.

Bará-Jiménez, S., Vicuña, P., Pineda, D., Henao, G. (2003). Perfiles neuropsicológicos y conductuales de niños con trastorno por déficit de atención/hiperactividad de Cali, Colombia. Revista de Neurología, 37(7): 608-615. Recuperado de https:// www.neurologia.com/articulo/2003189

Bayés, M., Ramos, J., Cormand, B., Hervás-Zuñiga, A., Del Campo, M., DuranTauleria, E., Ribasés, M., Vilella-Cuadrada, E., De Diego-Otero, Y., Casas-Brugué, M., Estivill, X. (2005). Genotipado a gran escala en la investigación del trastorno del espectro autista y el trastorno por déficit de atención con hiperactividad. Revista de Neurología, 40(1): 187-190. Recuperado de http://www.ub.edu/ geneticaclasses/brucormand/pdfs/33.pdf

Berenguer, C., Miranda, A., Pastor, G., Roselló, R. (2015). Comorbilidad del trastorno del espectro autista y el déficit de atención con hiperactividad. Estudio de revisión. Revista de Neurología, 60(1): 37-43. Recuperado de https://www.neurologia. com/articulo/2014559

Campeño-Martínez, Y., Gázquez-Linares, J., Santiuste-Bermejo, V. (2016). Medida de las emociones en niños con Trastorno por Déficit de Atención con Hiperactividad 
(TDAH). Un ensayo experimental con la técnica de potenciales evocados. UniversitasPsychologica, 15(5): 1-9. Doi: 10.11144/Javeriana.upsy15-5.ment

Capdevila-Brophy, C., Artigas-Pallarés, J., Obiols-Llandrich, J. (2006). Tempo cognitivo lento: ¿síntomas del trastorno de déficit de atención/hiperactividad predominantemente desatento o una nueva entidad clínica? Revista de Neurología, 42(2): 127-134. Recuperado de http://www.neurologia.com/pdf/Web/42S02/ uS02S127.pdf

Capdevila-Brophy, C., Artigas-Pallarés, J., Ramírez-Mallafré, A., López-Rosendo, M., Real, J., Obiols-Llandrich, J. (2005). Fenotipo neuropsicológico del trastorno de déficit atencional/hiperactividad: ¿existen diferencias entre los subtipos? Revista de Neurología, 40(1): 17-23. Recuperado de http://www.psyncron.com/wpcontent/uploads/2011/05/subtipostdah.pdf

Carrasco, X., Daiber, F., Rothhammer, P., Huerta, D., Andrade, C., Opazo, P., Lagos, L., Rothhammer, F., Aboitiz, F. (2012). Trastorno por déficit de atención e hiperactividad en niños aymara: primera aproximación clínica. Revista médica de Chile, 140(11): 1409-1416. Doi: 10.4067/S0034-98872012001100005

Conners, C., Sitarenios, G. (2011). Continuous Performance Test (CPT). Springer: New York.

De la Peña, F., Barragán, E., Rohde, L., Patiño, L., Zavaleta, D., Ulloa, R., Isaac, A., Murguía, A., Pallia, R., Larraguibel, M. (2009). Algoritmo de Tratamiento Multimodal para Escolares Latinoamericanos con Trastorno por Déficit de Atención con Hiperactividad (TDAH). Salud Mental, 32(1): 17-29. Recuperado de http:// www.medigraphic.com/pdfs/salmen/sam-2009/sams091c.pdf

Etchepareborda, M., Paiva-Barón, H. y Abad, L. (2009). Ventajas de las baterías de exploración neuropsicológica en el trastorno por déficit de atención/hiperactividad. Revista de Neurología, 48(2): 89-93. Recuperado de http://www.aidyne18.tizaypc. com/contenidos/contenidos/1/BateriaEvaluacion-ADHD.pdf

Fernandez-Mayoralas, M., Fernandez-Perrone, A., Munoz-Jareno, N., FernandezJaen, A. (2017). An update on the pharmacological treatment of attention deficit hyperactivity disorder: lisdexamphetamine and extended-release guanfacine. Revista de Neurología, 64(2): 1-8. Recuperado de https://www.ncbi.nlm.nih.gov/ pubmed/28272733

Galindo, M., Valera, V. (2016). Desempeño en el control inhibitorio en niños y niñas de 5 a 8 años con TDAH de la ciudad de Manizales. Repositorio, Universidad de Manizales. Manizales, Colombia. Recuperado de http://ridum.umanizales.edu. co:8080/xmlui/handle/6789/1903 
Golden, C. (2001). STROOP: Test de Colores y Palabras. Madrid, España: TEA Ediciones.

Grant, D., Berg, E. (2001). Test de Clasificación de Tarjetas de Wisconsin. Madrid, España: TEA Ediciones.

Guerrero, J., Pérez, R. (2011). El alumnado con TDAH (hiperactividad) como colectivo en riesgo de exclusión social: propuestas de acción y de mejora. Revista RUEDES, 1: 37-59. Recuperado de http://bdigital.uncu.edu.ar/objetos_digitales/3933/ guerrerogalanruedes2.pdf

López-Campo, G., Gómez-Betancur, L., Aguirre-Acevedo, D., Puerta, I., Pineda, D. (2005). Componentes de las pruebas de atención y función ejecutiva en niños con trastorno por déficit de atención/hiperactividad. Revista de Neurología, 40(6): 331-339. Recuperado de http://www.neurologia.com/pdf/Web/4006/s060331. pdf

López-Martín, S., Albert, J. Fernández-Jaén, A., Carretié, L. (2010). Neurociencia afectiva del TDAH: Datos existentes y direcciones futuras. Escritos de Psicología, 3(2): 17-29. Recuperado de http://scielo.isciii.es/scielo.php?script=sci arttext\&pid =S1989-38092010000100003

Martín-González, R., González-Pérez, P., Izquierdo-Hernández, M., HernándezExpósito, Alonso-Rodríguez, S., Quintero-Fuentes, I., Rubio-Morell, B. (2008). Evaluación neuropsicológica de la memoria en el trastorno por déficit de atención/ hiperactividad: papel de las funciones ejecutivas. Revista de Neurología, 47(5): 225230. Recuperado de http://www.neurologia.com/pdf/Web/4705/ba050225.pdf

Martínez, M., Hernández, N., Durán, X., Lluch, C., Fitó, A. (2017). Calidad de vida en niños con trastorno por déficit de atención con o sin hiperactividad (TDAH). Pediatría Atención Primaria, 19(73): 31-39. Recuperado de http://www.redalyc. org/pdf/3666/366650374005.pdf

Mateo, V., Vilaplana, A. (2007). Estrategias de identificación del alumno inatento e impulsivo desde el contexto escolar. Quaderns Digitals, 5, 13-28. Recuperado de http://www.quadernsdigitals.net/datos_web/hemeroteca/r_72/ nr_769/a_10377/10377.pdf

Mejía, C., Varela, V. (2015). Comorbilidad de los trastornos de lectura y escritura en niños diagnosticados con TDAH. Psicología desde el Caribe, 32(1): 121-144. Recuperado de http://www.redalyc.org/html/213/21337152005/

Miranda-Casas, A., Baixauli-Fortea, I., Colomer-Diago, C., Roselló-Miranda, B. (2013). Autismo y trastorno por déficit de atención/hiperactividad: convergencias y divergencias en el funcionamiento ejecutivo y la teoría de la mente. Revista 
de Neurología, 57(1): 177-184. Recuperado de https://www.neurologia.com/ articulo/2013279

Mulas, F., Gandía, R., Roca, P., Etchepareborda, M., Abad, L. (2012). Actualización farmacológica en el trastorno por déficit de atención/hiperactividad: modelos de intervención y nuevos fármacos. Revista de Neurología, 54(3): 41(53). Recuperado de http://www.neurologia.com/pdf/web/54s03/bhs03s041.pdf

Muñoz, J., Palau, M., Salvadó, B., Valls, A. (2006). Neurobiología del TDAH. Acta Neurológica Colombiana, 22(2): 184-189. Recuperado de http://www.acnweb. org/acta/2006_22_2_184.pdf

Ramos-Quiroga, J., Bosch-Munsó, R., Castells-Cervelló, X., Nogueira-Morais, M., García-Giménez, E., Casas-Brugué, M. (2006). Trastorno por déficit de atención con hiperactividad en adultos: caracterización clínica y terapéutica. Revista de Neurología, 42(10): 600-606. Recuperado de http://www.centrocade.com/wpcontent/uploads/2010/11/Trastorno..1.pdf

Ramos, C., Pérez-Salas, C. (2017). Control inhibitorio y monitorización en población infantil con TDAH. Avances en Psicología Latinoamericana, 35(1): 117-130. Doi: 10.12804/revistas.urosario.edu.co/apl/a.4195

Rico-Moreno, J., Tárraga-Mínguez, R. (2016). Comorbilidad de TEA y TDAH: revisión sistemática de los avances en investigación. Anales de Psicología, 32(3): 810-819. Doi: 10.6018/analesps.32.3.217031

Ríos-Flórez, J. A., Álvarez-Londoño, L. M., David-Sierra, D. E., Zuleta-Muñoz, A. C. (2018). Influencia del nacimiento pretérmino en procesos conductuales y emocionales de niños en etapa escolar primaria. Revista Latinoamericana de Ciencias Sociales, Niñez y Juventud, 16(1), 177-197. Doi: 10.11600/1692715x.16110

Ríos-Gallardo, Á, Hernández, A., Nieto, D., Losada, M., Bonilla-Santos, J., Cuéllar, L. (2017). Efectividad de una intervención cognitiva en funciones ejecutivas para mejorar flexibilidad mental en niños con trastorno por déficit de atención e hiperactividad. RFS, 8(1): 16-22. Recuperado de https://www.journalusco.edu. co/index.php/rfs/article/view/1328

Rodríguez-Jiménez, R., Cubillo, A., Jiménez-Arriero, M., Ponce, G., Aragüés-Figuero, M. Palomo, T. (2006). Disfunciones ejecutivas en adultos con trastorno por déficit de atención e hiperactividad. Revista de Neurología, 43(11): 678-684.Recuperado de http://www.centrocade.com/wp-content/uploads/2010/11/addh_func_ ejecutivas1.pdf

Román, A. (2014). Control inhibitorio en niños y niñas con TDAH medicados y no medicados que asisten a programas de atención a la población infantil en la ciudad de Manizales. Tesis de Maestría, Universidad de Manizales. Manizales, Colombia. 
Recuperado de http://ridum.umanizales.edu.co:8080/xmlui/bitstream/ handle/6789/2182/control\%20inhibitorio\%20en\%20ni\%C3\%B1os\%20y\%20 ni\%C3\%B1as\%20con\%20TDAH.pdf? sequence $=1 \&$ isAllowed $=\mathrm{y}$

Romero-Ayuso, D., Maestú, F., González-Marqués, J., Romo-Barrientos, C., Andrade, J. (2006). Disfunción ejecutiva en el trastorno por déficit de atención con hiperactividad en la infancia. Revista de Neurología, 42(5): 265-271. Recuperado de https://www.cop-cv.org/db/eventos/170131182854RyHzMZUwWixB.pdf

Roselló, B., Berenguer, C. (2016). Componentes del funcionamiento ejecutivo en niños con TDAH y niños con TEA de alto funcionamiento. Repositorio, Universidad de Alicante. Recuperado de http://rua.ua.es/dspace/handle/10045/63738

Ruiz-García, M., Gutiérrez-Moctezuma, J. Garza-Morales, S., De la Peña-Olvera, F. (2005). Trastorno por déficit de atención con hiperactividad. Actualidades diagnósticas y terapéuticas. Boletín médico del Hospital Infantil de México, 62(2): 145-152. Recuperado de http://www.scielo.org.mx/scielo.php?script=sci_ arttext\&pid=S1665-11462005000200009

Ruiz, L., Sicachá, M. (2015). Perfil clínico de niños y niñas entre 6 y 12 años diagnosticados con trastornos del aprendizaje tipo dislexia y su comorbilidad con trastornos de conducta-TDAH. Tesis de Especialización, Universidad Católica de Pereira. Pereira, Colombia. Recuperado de http://repositorio.ucp.edu.co:8080/ jspui/handle/10785/3280

Orjales, I. (2007). El tratamiento cognitivo en niños con trastorno por déficit de atención con hiperactividad (TDAH): revisión y nuevas aportaciones. Anuario de Psicología Clínica y de la Salud, 3: 19-30. Recuperado de http://institucional. us.es/apcs/doc/APCS_3_esp_19-30.pdf

Orozco, N., Zuluaga, J. (2015). Teoría de la mente en niños y niñas con trastorno por Défcit de Atención con Hiperactividad "TDAH". Revista Tesis Psicológica, 10(2), 136-148. Recuperado de http://www.redalyc.org/html/1390/139046451009/

Pacheco, J. (2015). Colorantes artificiales e hiperactividad. Innutrition. Recuperado de http://www.innutrition.mx/blog-innutricionando/colorantes-artificiales-ehiperactividad

Pineda, W., Puentes, P. (2013). Estudio ex-post facto de la teoría de la mente en niños escolarizados diagnosticados con trastorno por déficit de atención-hiperactividad. Tesis Psicológica, 8(2): 144-161. Recuperado de http://www.redalyc.org/ html/1390/139029743010/

Presentación, M., Siegenthaler, R., Jara, P., Miranda, A. (2010). Seguimiento de los efectos de una intervención psicosocial sobre la adaptación académica, emocional 
y social de niños con TDAH. Psicothema, 22(4): 778-783. Recuperado de http:// www.redalyc.org/html/727/72715515038/

Sáenz, G. (2005). Prevalencia del TDAH en escuelas primarias de Chihuahua delimitada por género. Tesis de Maestría, Universidad Pedagógica Nacional, México. Recuperado de http://200.23.113.51/pdf/22424.pdf

Sánchez, D., Guillén, J. (2017). Epidemiología del tratamiento farmacológico del trastorno por déficit de atención e hiperactividad (TDAH) en la Región de Murcia: diferencias por sexo, edad y lugar de residencia. Anales de Pediatría. Recuperado de http://www.sciencedirect.com/science/article/pii/S1695403317300814

Sánchez-Pérez, N., González-Salinas, C. (2013).30.12189 Ajuste Escolar del Alumnado con TDAH: Factores de Riesgo Cognitivos, Emocionales y Temperamentales. Electronic Journal of Research in Educational Psychology, 11(30): 527-550. Doi: http://www.redalyc.org/html/2931/293128257011/

Solis, V., Quijano, M. (2014). Rehabilitación neuropsicológica en un caso de TDAH con predominio impulsivo. Revista Chilena de Neuropsicología, 9(2): 67-71. Recuperado de http://www.redalyc.org/html/1793/179331012005/

Solovieva, Y., Lázaro, E., Quintanar, L. (2008). Mecanismos de los lóbulos frontales en niños preescolares con déficit de atención y niños normales. Acta Neurológica Colombiana, 24(2): 65-75. Recuperado de http://www.acnweb.org/ acta/2008_24_S2_64.pdf

Van, A., Gutiérrez, C., González, R., Ibáñez, M. (2008). Prevalencia de trastorno por déficit de atención con hiperactividad en estudiantes de escuelas de Bogotá, Colombia. Acta Neurológica Colombiana, 24(1): 6-12. Recuperado de http:// www.acnweb.org/acta/2008_24_1_6.pdf 